ISSN1027-5495. Functional Materials, 23, No.2 (2016), p. 183-190

\title{
Scintillation, phonon and defect channel balance; the sources for fundamental yield increase
}

\author{
A. Gektin', A. Vasil'ev ${ }^{2}$ \\ ${ }^{1}$ Institute for Scintillation Materials, STC "Institute for Single Crystals" \\ National Academy of Science of Ukraine, 60 Lenina Av., 61001 Kharkiv, \\ Ukraine \\ ${ }^{2}$ Skobeltsyn Institute of Nuclear Physics, Lomonosov Moscow State \\ University, Leninskie Gory 1(2), 119991, Moscow, Russia
}

Received January 27,2016

\begin{abstract}
The estimation of the fundamental limits of scintillation efficiency requires the analysis not only of electron channel of the energy transformation but also of the role of interplay between electron and phonon channels. The well-known factor beta which estimates the efficiency of electron-hole pair production is determined by the balance of these channels at the cascade and thermalization stages. The attention is paid to phonon production and relaxation at different stages of energy transformation in scintillators, starting from linear processes of emission of bulk phonons to multiphonon processes resulting in the production of local phonons and transient defect creation. The analysis shows that transient defects can play some positive role, being the centers of exciton stabilization. The spatial and temporal evolution of phonon subsystem and its interaction with electron subsystem in scintillators is analyzed. The role of phonons in the regions of high density of excitations is underlined. Possible experiments with simultaneous detection of these two channels are discussed. Keywords: scintillation efficiency, phonon, energy transformation.
\end{abstract}

Показано, что оценка сцинтилляционной эфрфективности требует анализа не только электронного канала преобразования энергии, но и взаимодействия между электронным и фононным каналами. Эффективность создания электронно-дырочных пар определяется балансом этих каналов при формировании каскада и при термализации возбуждений. Анализируются этапы преобразования энергии в сцинтилляторах, связанные с линейными процессами испускания объемных фононов и с многофононными процессами, а также с созданием фононов при образовании дефектов структуры. Анализ показывает, что дефекты могут играть определенную положительную роль, будучи центрами стабилизации экситонов. Анализируется пространственная и временная эволюция фононной подсистемы, а также ее взаимодействие с электронной подсистемой. Подчеркивается особая роль фононов в областях с высокой плотностью возбуждений.

Канали релаксації енергії і джерела підвищення сцинтиляційної ефективності. О. Гектін, А. Васильєв

Показано, що оцінка сцинтиляційної ефективності вимагає аналізу нетількиелектронного каналуперетворення енергії, алеівзаємодії між електроннимі фононним каналами. Ефективність створення електронно-діркових пар визначаеться балансом цих каналів при формуванні каскаду та при термалізації збуджень. Аналізуються етапи перетворення енергії в сцинтиляторах, пов'язані з лінійними процесами випускання об'ємних фононів і багатофононними процесами, а також 3 появою фононів при утворенні дефектів структури. Аналіз показує, що дефекти можуть грати певну позитивну роль як центри стабілізації екситонів. Аналізуеться просторова і тимчасова еволюція фононної підсистеми, а також її взаемодія з електронною підсистемою. Підкреслюеться особлива роль фононів в областях з високою щільністю збуджень. 


\section{Introduction}

The estimation of scintillation efficiency implies the analysis of the energy balance between useful and waste channels at each stage of energy transformation. Commonly used formula $\beta^{-1} S Q[1]$ shows rough factorization of the efficiency according to these stages: (1) production of cascade of electron-hole excitations ( $\beta=E_{e-h} / E_{e x}$, where $E_{e-h}$ is the mean energy for production of electronhole pair [1-3], $E_{e x}$ is the minimal energy of a neutral electronic excitation - an exciton or an electron-hole pair); (2) thermalization and energy transfer to emission centers ( $S$ ); and (3) namely the emission stage $(Q)$. Waste channels are connected with production of crystal vibrations of different types, namely bulk (delocalized) phonons and localized vibrations and distortions. The accurate estimation of the each stage efficiency requires the detail study of the vibrations occurence in ionic system and the balance of channels (see Fig. 1). Bulk phonons are produced in parallel with cascade stage and during thermalization, whereas local phonons are mainly generated during capture and emission stage. We discuss these channels in the following sections.

\section{Results and discussion}

\section{First stage - cascade}

The cascade stage involves both ionization of the matter by primary particle and secondary electrons (holes) of different energy. The duration of this stage is rather short (up to $10 \mathrm{fs}$ for cascade originated by $\mathrm{keV}$ electrons). This ionization is due to effect of electric field of moving particles on media. This field create polarization of the media at different frequencies, from low frequencies which polarize the lattice up to high frequencies which polarize electronic subsystem. At this stage the role of phonon emission is small. It can be estimated using the standard expression for the stopping power. The simplified expression for the stopping power can be written in non-relativistic form using the energy loss function $\operatorname{Im}\left(-\frac{1}{\varepsilon(\hbar \omega, \hbar \mathbf{q})}\right) \approx \operatorname{Im}\left(-\frac{1}{\varepsilon(\hbar \omega, 0)}\right)$ :

$$
-\frac{d E}{d x}=\frac{e^{2} m}{4 \pi \varepsilon_{0} \hbar^{2} E} \int_{0}^{E} \hbar \omega d(\hbar \omega) \operatorname{Im}\left(-\frac{1}{\varepsilon(\hbar \omega, 0)}\right) \ln \frac{\sqrt{E}+\sqrt{E-\hbar \omega}}{\sqrt{E}-\sqrt{E-\hbar \omega}} .
$$

The energy loss function is non-zero in all regions of photon energy which can be absorbed by the media. For insulators it includes fundamental absorption region $\hbar \omega>E_{e x}$ (an ionizing particle transfers a part of its energy to excitons, electron-hole pairs and core level excitations), possible absorption bands in the transparency region (where the transfer results in excited or ionized states of defects), and infrared absorption region with $\hbar \omega<\hbar \Omega_{\max }$ where phonons are emitted. Here $\Omega_{\max }$ is the maximal frequency of phonons (which is about longitudinal optical phonon frequency $\Omega_{L O}$ ). The integral in Eq. (1) is taken over all energies including phonon energy region from zero to the energy of ionizing particle. We can separate these two types of energy losses (neglecting the energy losses due to impact excitation/ionization of defects):

$$
\begin{gathered}
\left(-\frac{d E}{d x}\right)_{e-h}=\frac{e^{2} m}{4 \pi \varepsilon_{0} \hbar^{2} E} \int_{E_{e x}}^{E} \hbar \omega d(\hbar \omega) \operatorname{Im}\left(-\frac{1}{\varepsilon(\hbar \omega, 0)}\right) \ln \frac{\sqrt{E}+\sqrt{E-\hbar \omega}}{\sqrt{E}-\sqrt{E-\hbar \omega}}, \\
\left(-\frac{d E}{d x}\right)_{p h}=\frac{e^{2} m}{4 \pi \varepsilon_{0} \hbar^{2} E} \int_{0}^{\min \left(\hbar \omega_{\max }, E\right)} \frac{\hbar \omega}{1-e^{-\hbar \omega / k_{B} T}} d(\hbar \omega) \operatorname{Im}\left(-\frac{1}{\varepsilon(\hbar \omega, 0)}\right) \ln \frac{\sqrt{E}+\sqrt{E-\hbar \omega}}{\sqrt{E}-\sqrt{E-\hbar \omega}}- \\
\frac{e^{2} m}{4 \pi \varepsilon_{0} \hbar^{2} E} \int_{0}^{\hbar \hbar_{\max }} \frac{\hbar \omega}{e^{\hbar \omega / k_{B} T}-1} d(\hbar \omega) \operatorname{Im}\left(-\frac{1}{\varepsilon(\hbar \omega, 0)}\right) \ln \frac{\sqrt{E+\hbar \omega}+\sqrt{E}}{\sqrt{E+\hbar \omega}-\sqrt{E}}
\end{gathered}
$$

We include phonon absorption processes in Eq. 3. We do not need to use such term in Eq. 2 since thermal energy $k_{B} T<<E_{e x}$. The first term in Eq. 3 is greater than the second one for all energies $E$ greater than $\hbar \Omega_{\max }$, and therefore the stopping power is positive for these energies. Using this formula one can estimate the production of vibrations and distortions of ionic system in the perturbed region generated by the passage of energetic electrons. In order to do this we can use the sum rules for electronic and vibrational parts of energy loss function: 


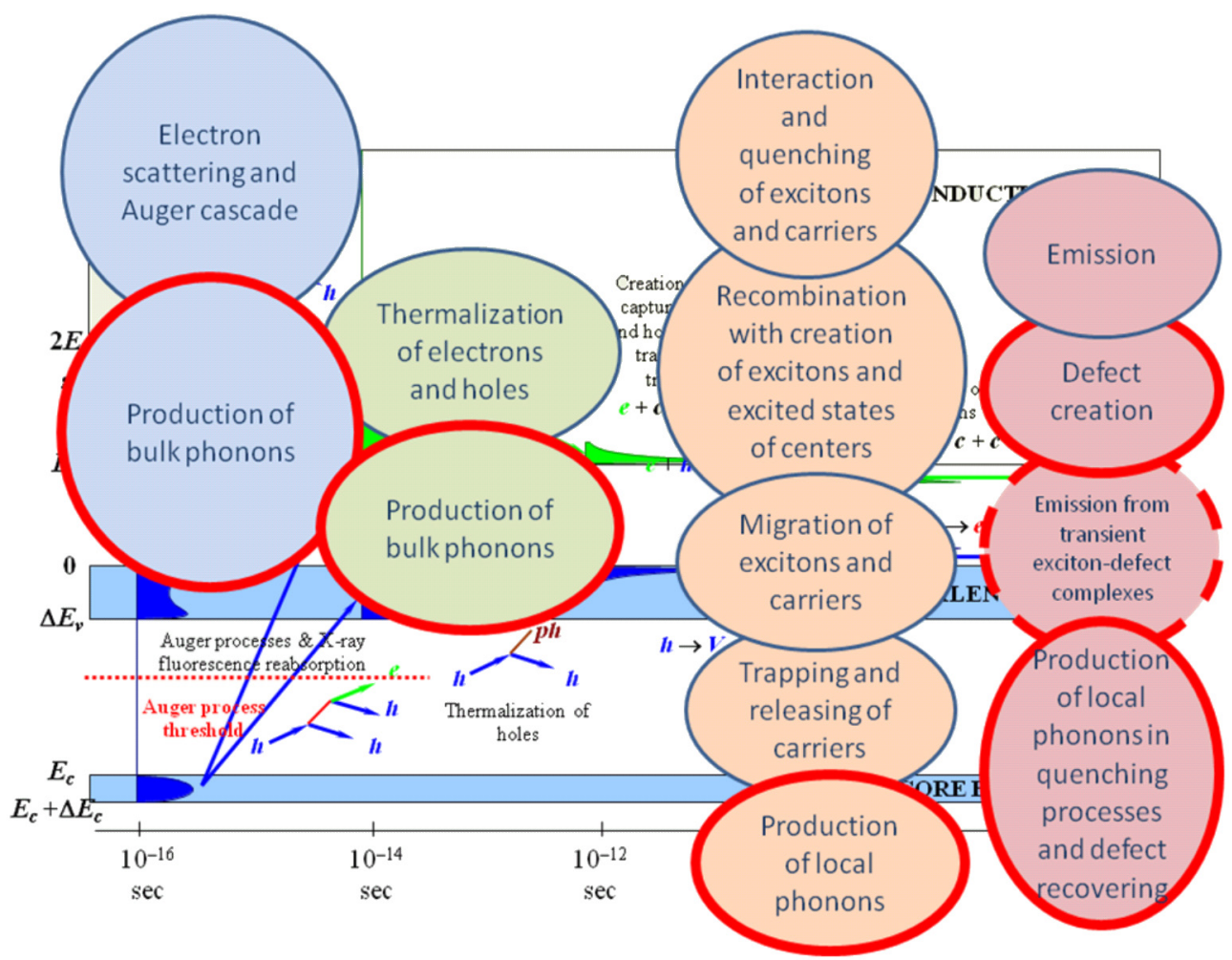

Fig. 1. Phonon processes during the energy transformation in scintillators.

$$
\begin{gathered}
\int_{\mathrm{W}_{L O}}^{E / \hbar} \omega d \omega \operatorname{Im}\left(-\frac{1}{\varepsilon(\omega)}\right)=\frac{\pi}{2} \omega_{p l}^{2}(E) \equiv \frac{\pi}{2} \frac{e^{2} N_{e f f}(E)}{\varepsilon_{0} m_{0}}, \\
\int_{0}^{\Omega_{L O}} \omega d \omega \operatorname{Im}\left(-\frac{1}{\varepsilon(\omega)}\right)=\frac{\pi}{2} \Omega_{L O}^{2} .
\end{gathered}
$$

Here $\hbar \omega_{p l}$ is the plasmon energy, which can be expressed through effective concentration of electrons $N_{\text {eff }}(E)$ involved in transitions with energy less than $E, m_{0}$ is free electron mass. Therefore rough estimation of the ratio of energy loss channels for primary electron with kinetic energy well above plasmon energy $\hbar \omega_{p l}$

$$
\frac{(d E / d x)_{\text {phonon }}}{(d E / d x)_{e-h}} \approx \frac{\Omega_{L O}^{2}}{\omega_{p l}^{2}(E)} \approx\left(\frac{(10-100) \mathrm{meV}}{(10-20) \mathrm{eV}}\right)^{2} \approx 10^{-6}-10^{-4}
$$

The account for $q$-dependence of energy loss function in Eq. (1) can describe not only relatively low-energy electronic excitations and lattice vibrations (phonons) with small momentum $\hbar \mathbf{q}$ but also much more rare events - impact production of fast electrons ( $\delta$-electrons) and recoil ions (very weak channel).

The stopping power with production of additional electron-hole pairs (Eq. 2) decreases to zero when the kinetic energy of moving charge approaches to $E_{e x}$. In semiconductors Keldysh formula is often used, $(d E / d x)_{e-h} \sim\left(E-E_{e x}\right)^{2}$ (this formula is applicable for $\left.E<\hbar \omega_{p l}\right)$ [4]. In crystals with strong excitonic effects the power is 1.5 instead of 2 , but again the stopping power smoothly increases above the threshold [5]. Therefore in some energy region of kinetic energies above $E_{e x}$ the inelastic scattering competes with relaxation with phonon emission, and the effective thresholds for production of secondary excitations $E_{t h}^{e(h)}>E_{e x}$. At the end of the cascade stage all electrons and holes have kinetic energies less than $E_{t h}^{e(h)}$ and therefore new electronic excitations cannot be produced any more. Electron and hole kinetic energies after the cascade stage are distributed with 

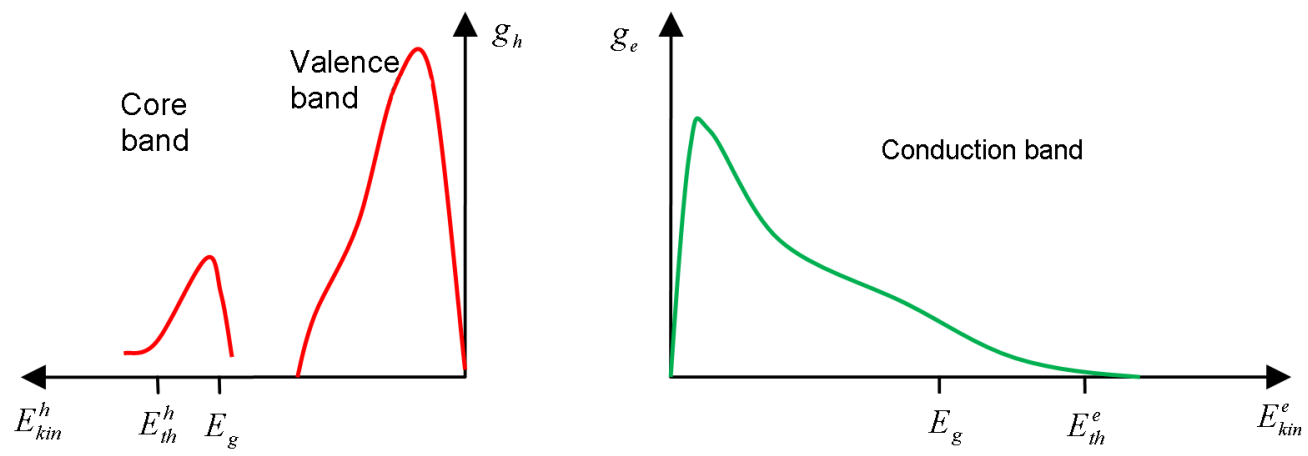

Fig. 2. The distribution of electrons and holes over their kinetic energies after the cascade of inelastic electron-electron scattering prior to thermalization. The case of crossluminescent crystal is shown, for which the top of the uppermost core band is below the hole threshold energy $E_{t h}^{h}$.

distribution functions $g_{e(h)}\left(E_{k i n}\right)$ from 0 to energies slightly more than $E_{t h}^{e(h)}$ (Fig. 2). These distribution are supposed to be normalized: $\int g_{e}\left(E_{k i n}^{e}\right) d E_{k i n}^{e}=1, \int g_{h}\left(E_{k i n}^{h}\right) d E_{k i n}^{h}=1$. The mean energy for creation of one electronic excitation is directly connected with this distribution:

$$
E_{e-h}=E_{g}+\int E_{\text {kin }}^{e} g_{e}\left(E_{k i n}^{e}\right) d E_{k i n}^{e}+\int E_{\text {kin }}^{h} g_{h}\left(E_{\text {kin }}^{h}\right) d E_{\text {kin }}^{h} \equiv \beta E_{g} .
$$

Therefore typical values of $\beta$ is about 2-3 (Fig. 3). The values of $\beta$ are above 3 for crystals with wide valence band and for narrow forbidden gap (in this crystals the relative difference between threshold energies $E_{t h}^{e(h)}$ and $E_{g}$ is large). On the contrary, $\beta$ is less than 2 when the valence band is narrow. Moreover, some features of electronic structure can additionally reduce $\beta$. In [6] we demonstrated that the shift of the position of the uppermost core level in row CsCl-CsBrCsl relative to the threshold of Auger process can significantly modify $g_{e}\left(E_{k i n}\right)$ and decrease $\beta$ : $\beta_{C s C l}>\beta_{C s B r}>\beta_{C S I}$. The analogous effect is connected with the position of the plasmon resonance and the threshold of multiplication of electronic excitations. The distribution of energy of secondary excitations is controlled by the energy loss function, and many electron-hole pairs are created with total energy about plasmo energy. If the plasmon energy is higher than the electron threshold energy $\left(\hbar \omega_{p l}>E_{g}+E_{t h}^{e}\right)$, most of these excitations in crystals with narrow valence band could produce additional low-energy electron-hole pair and thus reduce $\beta$. In the opposite case $\hbar \omega_{p l}<E_{g}+E_{t h}^{e}$ most of the electrons from the plasmon resonance peak could not produce additional pairs and remains hot until thermalization stage. In this case $\beta$ is also high.

\section{Second stage - thermalization}

At the thermalization (up to $10 \mathrm{ps}$ ) stage the interaction of moving charges with media is still described by the same formulas through the energy loss function, but at this stage the phonon channel (Eq. 3) is now the only channel of energy losses. During this stage the whole remain kinetic energy of electrons and holes should be transferred to phonons. The fraction $1-1 / \beta$ of the total deposited energy is emitted as phonons at this very thermalization stage, and this fraction can be large. Typical values of $\beta$ is about 1.5-3 (see Fig. 3), and therefore about 30 to $70 \%$ of energy is released as phonons. The main types of phonons, which are emitted by electrons with high kinetic energy, are long-wavelength longitudinal optical (LO) phonons.

The kinetics of phonons is often not discussed. Phonon mean free path is determined by phonon group velocity (for acoustical phonons it is equal to sound velocity at small phonon momentum, whereas for optical phonons it is usually much less than the sound velocity and is proportional to the phonon momentum). Lifetime of phonons is determined by the scattering processes, mainly phonon-phonon scattering due to anharmonicity (mainly splitting of phonon into two phonons, for instance low-momentum LO phonon is converted into two LA phonons with large opposite momenta). It is worth noting that normal processes result in conservation of total momentum, and therefore the main mechanism of dissipation is so-called umklapp processes, when the momen- 


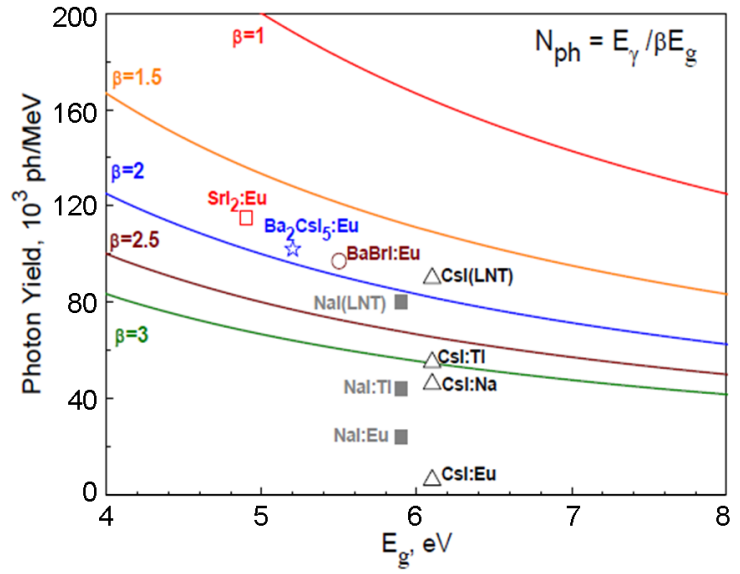

Fig. 3. Typical scintillator photon yields as a function of forbidden gap energies in the assumption of $\mathrm{S}=\mathrm{Q}=1$. Experimental values for some halide crystals are shown by symbols. tum can be preserved only with the account for additional basic vectors in the reciprocal space. Another important mechanism is phonon scattering on lattice defects and charge carriers. A typical phonon lifetime is about 1 to $100 \mathrm{ps}$, and the typical mean free path is about 1 to $100 \mathrm{~nm}$ (see, e.g. [7-9]). This mean free path is strongly temperature dependent, and at cryogenic temperatures the phonons are scattered mainly by crystal boundaries. These vibrationally excited regions can be especially important at the end of the track where the density of secondary electronic excitations is high, and along the whole track of heavy ionizing particles such as a-particles or hadrons. We can estimate the electric fields induced in the media by moving particle with the velocity $v$ using Lienard-Wiechert potential formulas [10]:

$$
\begin{gathered}
E_{z}(z, \rho, t) \sim-\frac{e \Omega_{L O}^{2}}{2 \pi \varepsilon_{0} \tilde{\varepsilon} v^{2}} K_{0}\left(\frac{\Omega_{L O} \sqrt{\rho^{2}+4 / q_{\max }^{2}}}{v}\right) \cos \left(z \Omega_{L O} / v-\Omega_{L O} t\right) \\
E_{\rho}(z, \rho, t) \sim \frac{e \Omega_{L O}^{2}}{2 \pi \varepsilon_{0} \tilde{\varepsilon} v^{2}} \frac{\rho}{\sqrt{\rho^{2}+4 / q_{\max }^{2}}} K_{1}\left(\frac{\Omega_{L O} \sqrt{\rho^{2}+4 / q_{\max }^{2}}}{v}\right) \sin \left(z \Omega_{L O} / v-\Omega_{L O} t\right),
\end{gathered}
$$

where $q_{\max } \approx \pi / a$ is the maximum wave vector transmitted to the lattice.

The radius of this vibrationally excited region can be estimated as $v / \Omega_{L O}$, where $v$ is the carrier velocity. For an electron with kinetic energy about $5 \mathrm{eV}$ and $\hbar \Omega_{L O}=0.1 \mathrm{eV}$ this radius is about $8 \mathrm{~nm}$. Typical spatial distribution of these electric field of LO vibrations directed along the track and perpendicular to the track for different phonon frequencies and different electron energies are shown in Fig. 4. These vibrations form the wake region after the travelling electron or hole. The total energy of the vibrations can be estimated using the expression

$$
2 \pi \tilde{\varepsilon} \frac{1}{2} \int_{0}^{\infty} \rho d \rho\left(E_{z}^{2}(z, \rho)+E_{\rho}^{2}(z, \rho)\right) .
$$

This total energy should be equal to phonon part of energy losses

$$
-\frac{d E}{d x}=\frac{\hbar^{2} \Omega_{L O}^{2}}{2 a_{B} \tilde{\varepsilon} E} \ln \frac{\sqrt{E}+\sqrt{E-\hbar \Omega_{L O}}}{\sqrt{E}-\sqrt{E-\hbar \Omega_{L O}}} .
$$

By the way, the electron-phonon interaction at these stages controls the efficiency of intraband luminescence which is regarded as one of the possible mechanisms of fast timing with the resolution of $\sim 10$ ps [11].

Some parameters of the vibrational wake region after the travelling particle for two typical phonon energies are presnted in Table. Here $E_{k i n}$ is the kinetic energy of the particle, $\langle\rho\rangle$ is the mean radius of the vibrating region, $\lambda_{L O}$ is the carrier mean free path with respect to LO phonon emission, $\Delta E$ is the local deposit of vibrational energy along the track $\left(\Delta E=\hbar \Omega_{L O} /\left(\pi\langle\rho\rangle^{2} \lambda_{L O}\right)\right.$ ), and the last column is the local deposit of this energy measured in Kelvin per $\mathrm{nm}^{3}$.

The case $\hbar \Omega_{L O}=0.1 \mathrm{eV}$ corresponds to oxides and fluorides. In this case the radius of the wake region is rather narrow for all kinetic energy of thermalizing charge carriers, and decreases from $3 \mathrm{~nm}$ for electrons with high kinetic energy (just below the threshold of inelastic scattering, which is about $E_{e x}$ ), to less than $1 \mathrm{~nm}$ at the end of the thermalization path. The mean free path for LO phonon emission is rather low at the end of the trajectory (where kinetic energy is about few phonon energies). Therefore we can say that in crystals with such phonon energies the wake region is formed by rather compact phonon wavepackets, and the overheating in this wake region is significant. 
Table. Some parameters of the wake region for different parts of thermalization trajectory

\begin{tabular}{|c|c|c|c|c|c|}
\hline$\hbar \Omega_{L O}, \mathrm{eV}$ & $E_{\text {kin }}, \mathrm{eV}$ & $\langle\rho\rangle, \mathrm{nm}$ & $\lambda_{L O}, \mathrm{~nm}$ & $\Delta E, \mathrm{meV} / \mathrm{nm} 3$ & $\Delta T, \mathrm{~K} / \mathrm{nm}^{3}$ \\
\hline 0.1 & 0.2 & 0.75 & 0.6 & 70 & 814 \\
\hline 0.1 & 1.0 & 1.8 & 1.5 & 12 & 142 \\
\hline 0.1 & 5.0 & 3.0 & 5.0 & 2 & 24 \\
\hline 0.01 & 0.1 & 3.8 & 1.4 & 0.5 & 6.6 \\
\hline 0.01 & 1.0 & 9.0 & 8.9 & 0.03 & 0.46 \\
\hline 0.01 & 5.0 & 17 & 35 & 0.005 & 0.06 \\
\hline
\end{tabular}
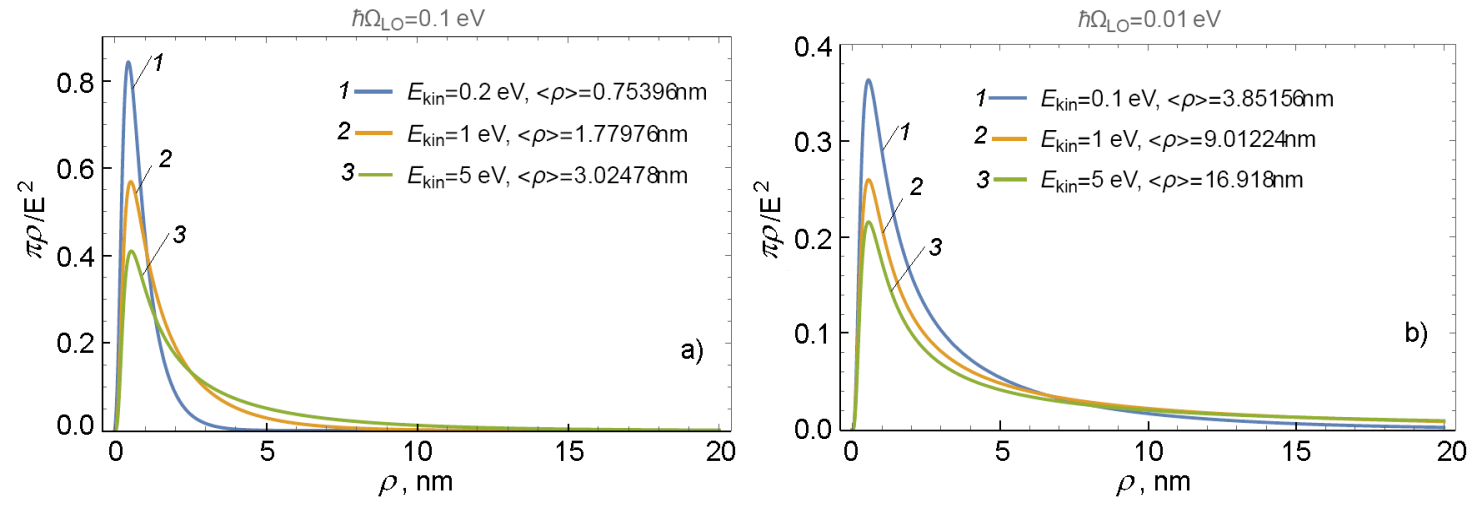

Fig. 4. Radial distribution of electric field in the wake region of a travelling particle for different electron kinetic energies and two values of phonon energy: $\hbar \Omega_{L O}=0.1 \mathrm{eV}$ (a) and $\hbar \Omega_{L O}=0.01 \mathrm{eV}$ (b).

The case $\hbar \Omega_{L O}=0.01 \mathrm{eV}$ corresponds to iodide crystals. In this case the wake region is much wider, mean free path is longer, and therefore the local volume deposit of vibrational energy is much less than for the previous case. Therefore the temperature increase in the wake region is much less than for the case of oxides.

High energy deposit at the end of the thermalization trajectory does not mean that a carrier is stopped in this region with high temperature. In case of binary crystals the thermalization stage is splitted into two regions - relaxation with emission of optical phonons is followed by the relaxation with emission of acoustical phonons. The energy deposit along this part of trajectory is small, and the total thermalization length can be rather long. Therefore a charge carrier can go away from the overheated region. This separation of the thermalization into two stages is typi-

cal only for binary crystals. In crystals with complicated phonon structure the total thermalization is connected with different optical phonons, and therefore carriers could reach thermal energy in overheated zone.

The typical rates of $\mathrm{LO} \rightarrow \mathrm{LA}+\mathrm{LA}$ transformation is from 5 to $50 \mathrm{ps}$ for GaAs [7]. Therefore the spatial expansion of the region with high intensity of optical vibrations occurs mainly in ballistic way at initial ten picoseconds. The spread velocity for acoustical phonons is equal to sound velocity (about $5 \mathrm{~nm} / \mathrm{ps}=5 \cdot 10^{5} \mathrm{~cm} / \mathrm{s}$ ), whereas the spread velocity of optical phonons is much less $\left(v_{L O}=d \Omega_{L O}(q) / d q<<c_{L A}\right.$, which is evident from the phonon energy dispersion law).

The consideration of the wake region could be useful not only for analysis of the vibrations in this region, but also could be useful in estimation of the distance from the trajectory of primary 

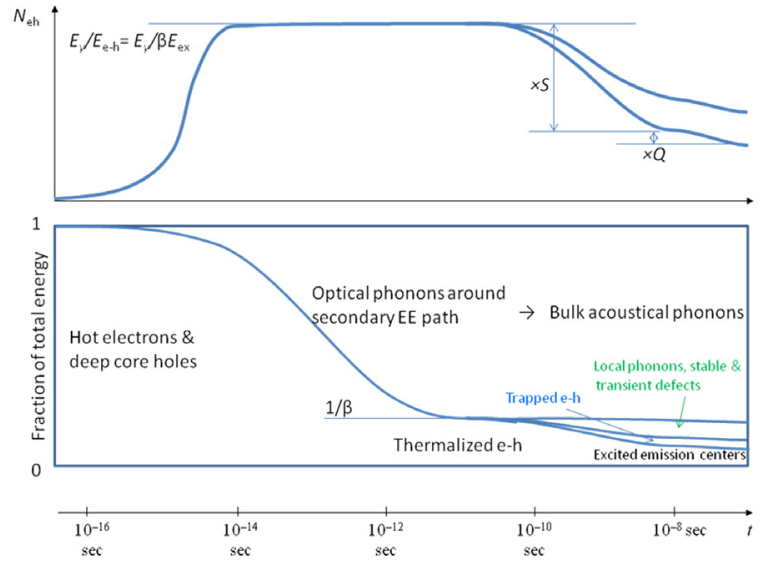

Fig. 6. Evolution of the mean number of electronic excitations and fraction of electron and phonon channels ionizing particle to the birthplace of secondary electronic excitations. Let us suppose that most of electronic excitations are created through the production of electron-hole pairs with energy close to plasmon resonance. Fig. 5 shows the spatial distribution of plasmon oscillations which correspond to the resonance creation of secondary electron-hole pairs for different energies of kinetic energy of ionizing particle. This figure demonstrate that secondary excitations are created in 0.5 to $3 \mathrm{~nm}$ distance from the trajectory of ionizing particle. This result is important in estimation of initial spatial distribution of secondary excitations.

\section{Last stage-emission centers}

The final stage - processes of recombination of electrons and holes, emission of excitons or radiative relaxation - also involves

strong interaction between electron and lattice subsystems. The main difference between this stage and two previous stages is that the velocity of electronic excitations becomes low and electron-lattice interaction is much higher. At this stage the linear approach to electron-phonon interaction is no longer applicable. Strictly speaking, the electrons and holes form polarons, i.e. charge carriers surrounded by lattice distortions and vibrations. Self-trapped holes are an example of the polarons of small radius with very heavy mass, when the displacement of surrounding ions is of the order of interatomic distances. At this stage only multiphonon approach can be applied. Production of stable and transient defects (for instance, F-H pairs) is typical for this stage. This production of large distortions is commonly regarded as negative phenomenon, resulting e.g. in temperature quenching and decrease of radiation hardness. Nevertheless, it could play a positive role in the production of new types of emission centers - e.g. transient defects, which can stabilize excitons. Fast intrinsic luminescence of CsI possibly can be explained by this stabilization of electronic excitations on defects, created in relaxation process of multiply ionized ions in the center of clusters of electronic excitations.

\section{Conclusions}

Figure 6 shows the evolution of the mean number of electronic excitations and fraction of electron and phonon channels with time discussed in the previous sections.

For comprehensive study of these vibrational channels we could propose a set of experiments with simultaneous detection of photon and phonon responses of scintillating crystals. We are not aware of such measurements considering both channels done before. Some information can be provided by bolometric experiments like those used for rare events search (double neutrino-less decay, dark matter search). Another way of indirect search for transient defect creation is the study of fast emission, which is connected with "radiation shake-off" studied by Gavrilov and Aluker in 80th [12]. This effect was studied in model alkali halides, but was not investigated in scintillating materials.

\section{Acknowledgements}

AV gratefully acknowledge a financial support of the RF Ministry of Education and Science under the Agreement RFMEFI61614X0006. 


\section{References}

1. A. Lempicki and A. J.Wojtowicz, J. Luminescence, 60-61, 942 (1994).

2. P. A. Rodny, Physical Processes in Inorganic Scintillators. Boca Raton, FL, USA: CRC Press, 1997, p. 219

3. P. Lecoq, A. Annenkov, A. Gektin, M. Korzhik, and C. Pedrini, Inorganic Scintillators for Detector Systems: Physical Principles and Crystal Engineering, Springer, Berlin, 2010, p. 251.

4. L.V.Keldysh, Sov.Phys. Zh. Exper. Teor. Fiz., 21, 1135 (1965).

5. A.N. Vasil'ev, Y. Fang and V.V. Mikhailin, Phys. Rev., B60, 5340 (1999).

6. A.N. Vasil'ev, A.V. Gektin, IEEE Trans. Nucl. Scie., 61, 235 (2014).

7. T.Luo, J. Garg, J. Shiomi, et al, Europhys. Lett., 101, 16001 (2013).

8. T. Feng and X. Ruan, J.Nanomater., 2014, 206370 (2014).

9. J.P. Freedman, J.H. Leach, E.A. Preble, et al., Sci..Rep., 3, 2963 (2013).

10. N.P. Kalashnikov, V.S. Remizovich, M.I. Ryazanov, Collisions of Fast Charged Particles in Solids, Gordon and Breach, New York, 1985.

11. P.Lecoq, M.Korzhik, A.Vasil'ev, IEEE Trans. Nucl. Sci., 61, 229 (2014).

12. E.Aluker, V.Gavrilov, S.Chernov et al, Sov. Fiz. Tverdogo Tela, 26, 321 (1984). 